tient compte du poids de la dalle, on voit que la résultante des pressions passe dans le tiers médian du contrefort et que celui-ci n'est nulle part soumis sur sa base à aucun effort d'extension; et cela aura lieu $a$ fortiori si $\beta>45^{\circ}$ Si la direction CA devient perpendiculaire au parement amont, la poussé $P$ coupe la hase $B C$ juste au tiers amont. On concolt don facilement qu'il est toujours possible de donner au barrage un profil tel que la résultante des pressions en charge passe exactement par le millieu de la hasc, de manière à avoir une pression uniformément répartie sur cette base, desideratum vers lequel on doit tendre lorsqu'on a affaire à des terrains très peu résistants.

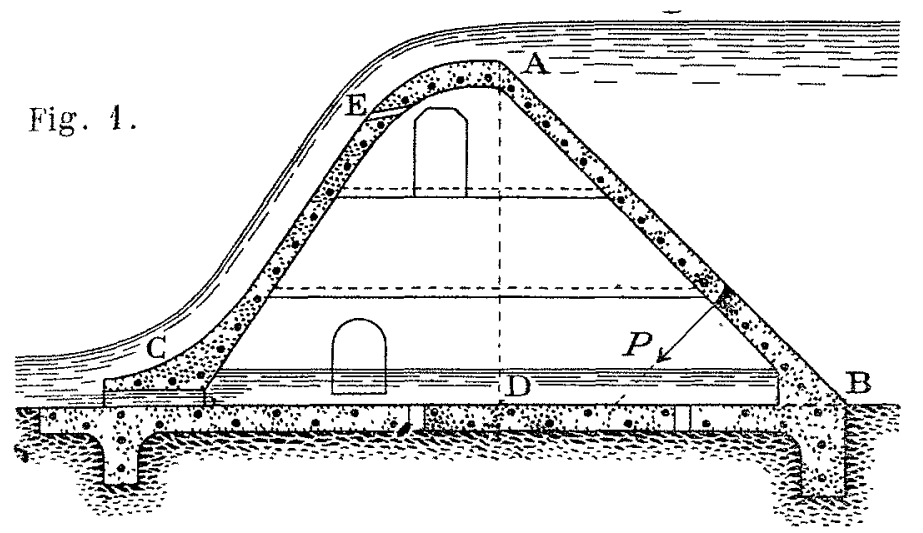

Lorsqu'un pareil barrage doit être fondé sur graviers, ou en général sur terrains peu résistants, les contreforts doivent s'appuyer sur un radier général en ciment armé, qui répartit la pression sur toute la longueur de l'ouvrage. Ce radier doit être prolongé un peu à l'aval, de manjière à éviter los aflouillements que produirait la lame déversante. De distance en distance, ce radier est percé de trous, pour laisser passer les eaux d'infiltration et éviter ainsi toute sous-pression.

Pour calculer la dalle AC du parement amont, on la divi. sera en un certain nombre de bandes horizontales, de faible hautcur, que l'on calculera comme une poutre encastrée reposant sur plusieurs appuis. Quant aux contreforts, on emploiera pour eux la mème méthode de calcul que pour les barrages pleins en maçonnerie.

Un pareil type de barrage coute moins cher qu'un barrage ordinaire en maçonnerie, aussi son emploi pourra sans doute permettre dans certains cas de rendre financièrement praticable l'installation de petites chutes jusqu'ici inutilisées; déjà les Américains parlent de construire des barrages de ce type de $50 \mathrm{~m}$. de hauteur.

Comme exemple de ce type d'ouvrage, nous signaleronsi ci le harrage de Schuylerville, dans l'Etat de New-York, dont le prolil est très sensiblement celui de la fig. 1, et qui a été construit avec une remarquable rapidité : commencé le 27 septembre 1904, il a été en effet terminé le 31 décembre de la mêne année. Sa hauteur est de $8 \mathrm{~m}$. 50 et sa longeur de $75 \mathrm{~m}$. La dalle AC du parement amont est inclinée à $45^{\circ}$ et se termine par une fondation, faite dans le rocher, de $1 \mathrm{~m} 50$ de largeur sur $0 \mathrm{~m} .90$ de profondeur. Les contreforts, ainsi d'ailleurs que la base du parement aval, reposent simplement sur le sol; la seule précaution qu'on ait prise a consisté dans un simple lavage du rocher au moyen d'un jet d"eau sous pression. Des ouvertures ont été ménagées à la base du parement aval pour laisser écouler les eaux d'inflitration; en outre, on a établi en $\mathrm{E}$, à la partie supérieure, des orifices de $75 \mathrm{~mm}$. de diamètre, dont le but est d'admettre de l'air sous la lame déversante et de rompre le vide partiel qui tend à se former ; on évite ainsi lout
" tremblement» du barrage. Une passerelle, s'appuyant sur les contreforts, est établie à l'intérieur du barrage; elle constitue d'ailleurs le seul moyen de communication qui existe entre les deux rives. Les contreforts sont espacés de $2 \mathrm{~m}$. 40 a $3 \mathrm{~m}$., et leur épaisseur est de $0 \mathrm{~m}$. 45 à la base et de $0 \mathrm{~m} .30$ au sommet. A la crête, le Jarrage à $0 \mathrm{~m} .60$ d'épaisseur, et un disposilif de hausses mobiles permet de surélever le niveau de l'eau. L'ouvrage a été calculé avec une lame d'eau de $1 \mathrm{~m} .50$, el nulle part il ne travaille à plus de $5,4 \mathrm{kgs}$ par centimètre carré.

Les Américains ne se contentent pas seulement de construire des barrages en ciment armé, ils y logent aussi leurs usines hydrauliques, témoin lusine d'oakdale, à Tippecanoe, dans l'Indiana, qui est schématiquement représentée par la figure 2 ci-jointe. (Figure extraite d'une conférence faite au Frankin Institute, de Philadelphie, et publiée dans le numéro de janvier 1906 du Journal de cette Socrété). Cette usine a $84 \mathrm{~m}$. de longueur sur $25 \mathrm{~m}$. de largeur, et elle comporte 5 groupes électrogènes, de 1000 lillowatts chacun, composés d'un alternateur produisant du courant à 3000 volts et actionné par une turbine double, à aspiration, fonctionnant sous $14 \mathrm{~m}$. de chute moyenne.

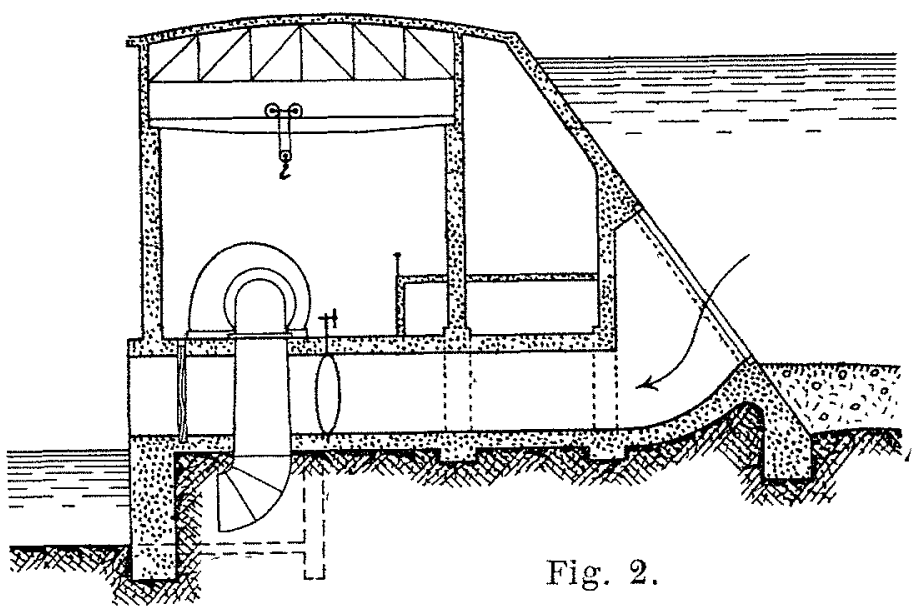

Ce dispositif d'installation hydro-électrique, qui dérive du type de l'usine-barrage construite sur une dérivation du Rhône à Cusset, près de Lyon, semble bien être le plus simple qu'il soit possible d'imaginer. Toutefois, nous nous demandous si le matériel électrique se trouvera bien de l'humidité qui règnera certainement dans une pareille usine. En tous cas. il y a là une curieuse expéricnce, que nous livrons à la méditation de nos bons chutards de ce côté de l'océan.

I. Bellet.

\section{Sur la Transmission des Courants allepnatifs}

Communications de M. Bronder à l'Académie des Sciences Séances des 7 mai et 25 jum 1906.

Application du principe de la superposition a fa transmission des courants alternatifs sur une longue ligne. Représentation graphique.

Si l'on appelle $U$ et $I$ les vecteurs représentatifs, en grandeur et en phase, de la tension et du courant au point $x$ d'un conducteur de la ligne $\left(^{*}\right), j$ le symbole imaginaire $\checkmark-I$, les équations différentielles du problème:

(") Quel que soit le nombre de phases des courants alternatifs à transmettre, on peut considérer isolément chaque conducteur de la ligne, en supposant celle-ci complétée fictivement par un conduc- 


$$
\begin{aligned}
& \frac{\mathrm{d} i}{\mathrm{~d} x}=g u+c \frac{\mathrm{d} u}{\mathrm{~d} t} \\
& \frac{\mathrm{d} u}{\mathrm{~d} x}=r i+l \frac{\mathrm{d} i}{\mathrm{~d} t}
\end{aligned}
$$

admettent, comme on le sait $\left(^{* *}\right)$, pour intégrales générales :

$$
\begin{gathered}
U=A e^{(a+b j) x-j 0}+B e^{-(a+b j) x-j 0^{\prime}} \\
I=\frac{A}{m} e^{(a+b j) x-j(0-\gamma)-\frac{B}{m} e-(a+b j) x-j\left(0^{\prime}-\gamma\right)}
\end{gathered}
$$

en désignant par $A, B, 0$ et $\theta$ ' des constantes d'intégration ; par $a$ et $b$ deux coefficients dépendant seulement de $r, g, l$, $e$ et $\omega$; et en posant :

$$
m=\sqrt{\frac{a^{2}+b^{2}}{g^{2}+\omega^{2} c^{2}}} \quad \operatorname{tg} \gamma=\frac{\omega c a-b g}{\omega c b+a g}
$$

Je me propose d'appliquer ici le principe de la superposition des solutions, qui résulte de la forme des équations (1) et (2), à la décomposition de chaque harmonique en un certain nombre de termes composants, commodes pour la pratique.

On prendra comme origine des abscisses $x$ de la ligne le point d'arrivée : le régitme à l'arrivée est défini, pour la fréquence considérée, par les amplitudes et les phases de la tension $U_{1}$ et du courant total $I_{1}$ débité dans les organes récepteurs. Nous considérerons séparément les deux solutions partielles correspondant respectivement à l'hypothèse d'une tension égale à $U_{1}$, combinée avec un courant nul (marche à vide), et d'un courant égal en grandeur à $I_{1}$, combiné avec une tension nulle (débit sur court-circuit).

Dans la première hypothèse $\left(I_{1}=0\right)$, on a évidemment $A=B=\frac{U_{1}}{2} ;$ les équations (3) et (4) prennent alors la forme :

$$
\left\{\begin{aligned}
U & =\frac{U_{1}}{2}\left(e^{a x}+e^{-a x}\right) \cos . b x+j \frac{U_{1}}{2}\left(e^{a x}-e^{-x x}\right) \sin . b x \\
& =\left(U_{1} \cosh \cdot a x \cos . b x+j U_{1} \sinh . a x \sin . b x\right) \\
J & =\left(\frac{U_{1}}{m} \sinh . a x \cos . b x+j \frac{U_{1}}{m} \cosh . a x \sin . b x\right) e^{j y}
\end{aligned}\right.
$$

La première représente la tension répartie à vide, la seconde, le courant de capacité réparti à vide. Dans chaque second membre entre crochets, le terme réel peut être considéré comme l'abscisse, et le coefficient de $j$ comme l'ordonnée d'un point du lieu de l'extrémité du vecteur $U$ ou $J$ correspondant, tracé à partir de $O$, en remarquant seulement que les axes $\mathrm{X}^{\prime} \mathrm{O} \mathrm{Y}^{\prime}$, auxquels est rapporté le lieu de $J$ (que j'appellerai courbe $\mathrm{N}$ ), doivent être tournés de

teur de retour de résistance nulle, parcouru par un courant nul. J'appelle donc: $r, l, z$, la résistance, la selt-induction et l'impédance par unité de longueur (en pratique le kilomètre) d'un des conducteurs utiles de la ligne; $c$ et $g$ sa capacité et sa " perditance" (coefficient de fuite ou conductance de perte), par unité de longueur, par rapport au conducteur de retour fictit; $;$ la pulsation des courants alternatıfs (ou plutôt de leur harmonıque considéré), u la tension par rapport au conducteur de retour au point $x ; i$ l'intensité au ni ẑme point, au temps $t$; on pose

$$
u=U e^{\jmath \omega t} ; \quad i=I e^{\jmath \omega t} .
$$

Les coefficients $a$ et $b$ qui figurent dans les solutions sont déterminés par l'égalıté connue :

$$
(a+b j)^{2}=r g-\omega^{2} c l+j \omega(r c+l g) .
$$

(**) Bulletin de la Societé Internationale des Electriciens, 5 avril 1905 , p. $311 \cdot 317$. l'angle $+\gamma$ par rapport aux axes $\mathrm{X}_{0} \mathrm{O} \mathrm{Y}_{0}$ du lieu de $U$ (que j'appellerai courbe $\mathrm{M}_{2}$ ).

On calcule aisément les coordonées des courbes en fonc* tion de $x$ au moyen de la Table 14 (fonctions hyperboliques et circulaires) du Recueil de M. Houel.

Dans la seconde hypothèse (intensité à l'arrivée $I_{1}$ nulle), on trouve de même $A=B=\frac{I_{1}}{2}$, d'où l'on déduit, par les équations (3) et (4). les nouvelles valeurs des vecteurs du courant utile $I$ et de la chute de tension correspondante produite par l'indépendance $V$.

$I=\left(I_{1} \cosh . a x \cos . b x+j I_{1} \sinh . a x \sin . b x\right) e^{-j \varphi_{1}}$ $V=\left(m I_{1} \sinh . a x \cos . b x+j m I_{1} \cosh . a x \sin . b x\right) e^{-j\left(\eta_{1}+\eta\right)}$

Comme plus haut, ces expressions indiquent les coordonées des lieux des extrémités des vecteurs (que j'appellerai les courbes $\mathrm{P}$ et $\mathrm{Q}$ respectivement) par rapport à deux systèmes d'axes rectangulaires $\mathrm{XOY}$ et $\mathrm{X}^{\prime \prime} \mathrm{OY}$ ", respectivement tournés de l'angle of et de l'angle $\left(0_{1}+\gamma\right)$ en retard sur $\mathrm{X}_{0} O \mathrm{Y}_{0}$.

Chaque régime est donc caractérisé par une épure à deux courbes (tension et courant) et la superposition des régimes se traduit par une superposition géométrique de deux épures. S'il n'y a pas de décalage de phase à l'arrivée entre $U_{1}$ et $I_{1}\left(\varphi_{1}=0\right)$, l'axe $O X$ du courant $I_{1}$ coïncide avec l'axe $\mathrm{OX}$ de la tension $U_{1}$ et les axes $\mathrm{X}^{\prime \prime} \mathrm{O} \mathrm{Y}^{\prime \prime}$ sont symétriques de X'OY' par rapport à $O X_{0}$; pour tenir compte d'un décalage quelconque o ${ }_{1}$, il suffir done de faire tourner du mêtme angle l'axe $\mathrm{OX}$ de la seconde épure par rapport à l'axe $\mathrm{OX}_{0}$ de la première. On obtient les tensions résultantes de $U$ et de $V$ en joignant deux à deux les points des extrémités des courbes de même espèce $M$ et $Q$, et les vecteurs des courants résultants de $l$ et de $J$ en joignant les points correspondants des courbes $\mathrm{N}$ et $\mathrm{P}$. De simples mesures sur l'épure font connaître en tous points de la ligne les grandeurs des tensions et des courants, leurs phases relatives, la puissance au départ $U_{0} I_{0} \cos \varphi_{0}$, la puissance à l'arrivée $U_{1} I_{1} \cos \varphi_{1}$ et, par suite, le rendement de la transmission.

\section{Etude simplifiée des effets de capacité}

Dans une précédente Note, j'ai montré comment le régime des courants alternatifs, en chaque point de la ligne: peut être obtenu par la superposition de deux épures relatives respectivement à la marche à vide et au fonctionnement en court-circuit. J'ai représentè ce dernier dans deux hypothèses : l'une correspondant à un décalage nul du coutrant à l'arrivée (courbe $\mathrm{P}$ des $I_{1}$ tracée en traits pleins, l'autre à un décalage $\varphi_{1}=3 \tau^{0}\left(\cos \varphi_{1}=0,80\right)$ du même courant (courbe $\mathrm{P}_{\varphi_{1}}$ des $I_{1}$ en traits pointillés). Les courbes $Q_{\varphi_{1}}$ et $P_{\varphi_{1}}$ sont obtenues simplement en faisant tourner de $37^{\circ}$ les axes XOY.

L'épure se rapportait à une transmission triphasée projetée de $1000 \mathrm{~km} s$, débitant à l'arrivée un courant $I_{1}$ de I oo ampères par phase, à 25 périodes, sous une tension étoilée $U_{1}$ de 60 ooo volts (correspondant à 103 goo volts entre fils). Les trois conducteurs câblés de la ligne ont chacun $83 \mathrm{~mm}^{2}$, sous I $1,6 \mathrm{~mm}$. de diamètre extérieur; leur écartement est de $2^{\mathrm{m}} 50$, et leur hauteur moyenne de $6 \mathrm{~m}$. au-dessus du sol. Les constantes kilométriques de chaque conducteur sont: $r=0,215 \mathrm{ohm} ; l=1,2632$ milli-henry: $c=0,29257 \mathrm{ohm} ; g$ a été pris égal à o, vu sa faible valeur, et à défaut de chiffres d'expériences. On en a déduit: $a=0,000229605 ; b=0,000524334 ; m=51 \mathrm{l}, \mathrm{I} 2 ;$ 
$\gamma=23038$ '55". On lit sur l'épure toutes les valeurs intéressantes des tensions et des courants, obtenus par exemple tous les $100 \mathrm{kms}$. Notamment au point $1000 \mathrm{kms}$ (départ), on obtient le régime à fournir par l'usine génératrice :

Pour $\cos \varphi_{1}=1 \quad$ Pour $\cos \varphi_{1}=0,80$

Tension résultante en volts Courant résultant en ampères Projection de $I_{0}$ sur $U_{0}$ ou $U_{0}^{\prime}$ Facteur de pussance cos $\varphi_{0}$, Puissance $U_{0} I_{0} \cos \varphi_{0}$, en kws Pussance $U_{1} I_{1} \cos \%_{1}$, en kws Rendenent de la transmission

$\begin{array}{ll}U_{0}=77800 & U_{0}=81600 \\ I_{0}=115 & I_{0}=78 \\ \text { amp. }=107,5 & \text { amp. }=77,5 \\ 0,93 & 0,99 \\ 25090 & 18972 \\ 18000 & 14400 \\ 0,72 & 0,76\end{array}$

Vecteur $\mathrm{ON}:=\frac{U_{1}}{m} \sqrt{\frac{\cosh \cdot 2 a x-\cos .2 b x}{2}}$

Vecteur $\mathrm{OQ} \mathrm{Q}_{1}:=m I_{1} \sqrt{\frac{\cosh \cdot 2 a x-\cos \cdot 2 b x}{2}}$

Je n'indique que les expressions utiles pour les calculs usuels. Quant au vecteur $O \mathrm{P}_{\mathrm{p}_{1}}$ et à ses projections, les expressions sont les mêmes que pour $\mathrm{OM}$ en remplaçant $U_{1}$ par $I_{1}$.

A ces expressions, il est intéressant pour les applications d'ajouter celles des angles suivants :

On remarquera sur l'épure que lit courbe $\mathrm{O} N$ (pour $g=0$ ) s'écarte peu de sa tangente à l'origine, perpendiculaire à OX; de même la courbe OQ s'écarte peu de la forme rectiligne et sa tangente à l'origine fait, avec $\mathrm{OY}$, un angle égal à $2 \%$.

Cela m'a conduit à rechercher d'une manière plus générale, pour $g>0$, des expres. sions approchées des différents vecteurs figurant dans l'épure et de leurs projections intéressantes, sous forme de courtes séries ordonnées par rapport aux valeurs croissantes de la distance $x$, prise comme variable. En posant pour simplifier

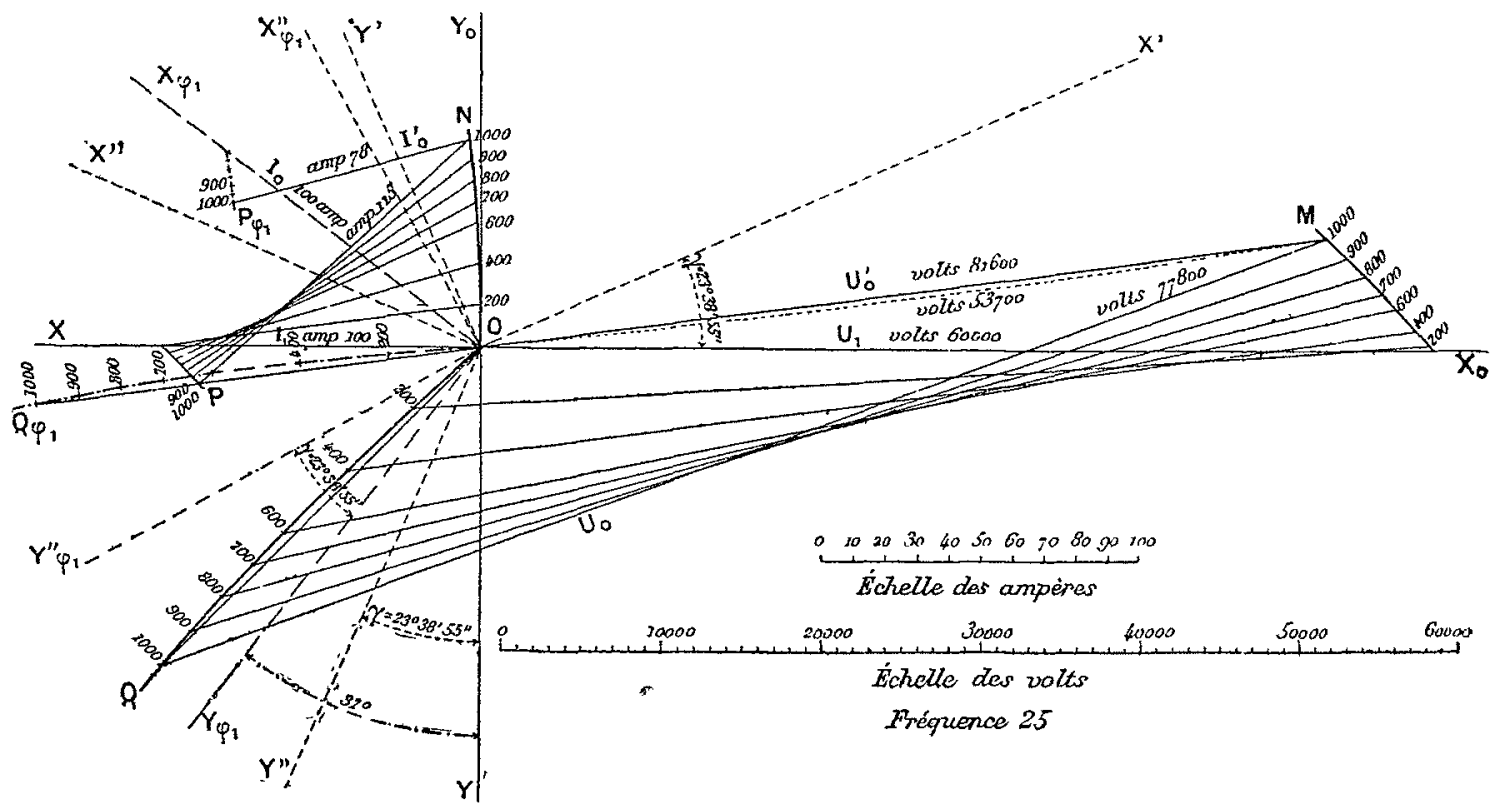

$$
\begin{aligned}
& p=\omega^{2} l c-g r \\
& q=\omega c r+\omega l g
\end{aligned}
$$

(I) ANGLES

VALEURS EXACTES

VALEURS APPROCHEES

j'ai trouvé les expressions suivantes :

\section{VALEURS APPROCHÉES}

Vecteur..... OM $=U_{1}\left(\mathrm{I}-\frac{p}{2} x^{2}\right)+\cdots \cdots$

Projection sur $\mathrm{OX}_{0}=U_{1}\left(\mathrm{r}-\frac{p}{2} x^{2}\right)+\cdots \cdots$

Projection sur $\mathrm{OY}_{0}=U_{1}\left(1-\frac{p}{6} x^{2}\right) \frac{q}{2} x^{2}+\cdots \cdots$

Vecteur...... ON $=U_{1} x\left(\mathrm{I}-\frac{p}{6} x^{2}\right) \sqrt{g^{2}+\omega^{2} c^{2}}+\cdots$

Projection sur $\mathrm{OX}_{0}=U_{1}\left(g x-\frac{p g+q \omega c}{6} x^{3}\right)+\cdots$

Projection sur $O Y_{0}=U_{1}\left(\omega c x-\frac{\omega p-g p}{6} x^{3}\right)+\cdots$

Vecteur...... OQ $Q_{q_{1}}=I_{1} z x\left(1-\frac{p}{6} x^{2}\right)+\cdots \cdots$

Projection sur $\mathrm{OX}_{\varphi 1}=I_{1}\left(r x-\frac{p r+q \omega l}{6} x^{3}\right)+\cdots \cdot$

Projection sur $\mathrm{OY}_{\varphi_{1}}=I_{1}\left(\omega l x-\frac{\omega l p-r q}{6} x^{3}\right)+$

\section{VALEURS EXACTES}

Vecteur OM : $=U_{1} \sqrt{\frac{\cosh \cdot 2 a x+\cos .2 b x}{2}}$
(2)

MOX $_{0}=\operatorname{arctg} \cdot \operatorname{tgh} . a x \operatorname{tg} . b x=\operatorname{arctg} \cdot\left[\frac{q}{2}\left(1+\frac{p}{3} x^{2}\right) x^{2}\right]+\cdot \cdot$ $\operatorname{NOP}_{\varphi_{1}}=\pi-\operatorname{arctg} \cdot \frac{\sin .2 b x}{\sinh .2 a x}-q_{1}-\gamma$

$\operatorname{MOQ} \varphi_{\varphi_{1}}=\pi-\operatorname{arctg} \cdot \frac{\sin .2 b x}{\sinh .2 a x}+\varphi_{1}+\gamma$

$\left.\begin{array}{|}\mathrm{XOQ} \\ \mathrm{X}_{\phi_{1}} \mathrm{OQ}_{\phi_{1}}\end{array}\right\}=\operatorname{arctg} \cdot \frac{\operatorname{tg} \cdot b x}{\operatorname{tgh} \cdot a x}-\gamma=\operatorname{arctg} \cdot \frac{6 \omega l-(\omega l p-r q) x^{2}}{6 r-(p r+\omega l g) \cdot x^{2}}+\cdots$ $\mathrm{NOX}_{0}=\operatorname{arctg} \cdot \frac{\operatorname{tg} \cdot b x}{\operatorname{tgh} . a x}+\gamma=\operatorname{arctg} \cdot \frac{6 \omega c-(\omega c p-g q) x^{2}}{6 g-(g p+\omega c q) x^{2}}+\cdot \cdot$

Ces valeurs des vecteurs et des angles permettent d'effectuer rapidement les calculs des vecteurs résultant par les formules :

$$
\begin{aligned}
& {\overline{Q_{\varphi_{1}}}}^{2}={\overline{Q_{\varphi_{1}}}}^{2}+\overline{\mathrm{OM}}^{2}+2 \overline{\mathrm{OQ}}_{\bar{q}_{1}} \overline{\mathrm{OM}} \cos Q_{\psi_{1}} \mathrm{OM} \\
& {\overline{\mathrm{P}_{\varphi_{1}}}}^{2}={\overline{\mathrm{P}_{\varphi_{1}} \mathrm{O}}}^{2}+\overline{\mathrm{ON}}^{2}-2 \overline{\mathrm{OP}}_{\varphi_{1}} \overline{\mathrm{ON}} \cos \mathrm{NOP}_{\varphi_{1}}
\end{aligned}
$$

Elles permettent aussi de remplacer dans les calculs le vecteur $O Q$ par ses projections, en remplaçant simplement les valeurs $r x$ et $\omega l x$, qu'auraient la résistance $\in t$ l'inductance pour une ligne sans capacité, par les valeurs corrigées des équations (Io) et (I I). Le calcul graphique des effets d'impédance des lignes présentant de la capacité peut alors se faire, au moyen de ces corrections de $r$ et de w $l$, comme pour les lignes sans capacité, en ajoutant en outre, au vecteur $U_{1}$ de la tension à l'arrivée, un petit segment correctif $\mathrm{X}_{0} \mathrm{M}$ dont les projections se déduisent des valeurs données plus haut pour les projections de $O M:(4)$ et (5). 
Ces formules approchées, qui dispensent des calculs de $a$ et $b$ et de l'emploi des fonctions hyperboliques, donnent des résultats très suffisamment exacts pour toutes les lignes aériennes ordinaires dont la longueur ne dépasse pas $500 \mathrm{kms}$ et pour les lignes souterraines usuelles jusqu'à bo kms. Au delà, il vaut mieux employer les formules complètes, ou les expressions plus simples données dans ma précédente communication et rapportées à des axes auxiliaires.

\section{LE MOIS HYDRO-ÉLECTRIQUE}

\section{ACADÉMIE DES SCIENCES}

\section{MÉCANIQUE ET ÉLECTRICITÉ}

Galvanomètre à cadre mobile pour courants alternatifs. Note de M. Henri AbraHaM, séance du 30 avril 1906.

On peut mesurer des courants alternatifs de l'ordre du centiène de micro-ampère avec un galvanometre à cadre mobule dont le champ magnétıque est crée par un électro-aimant excité par un courant alternatıf de même fréquence. Pour les mesures très délicates, 1 peut être bon d'actionner cet électro-amant au moyen d'un petst transformateur auxiliaire bier isolé.

Description de l'appareil. - L'apparesl a été réalısé avec la collaboration de IV. J. Carpentier, et nous avons aussi entrepris la construction d'un modèle mons sensible destmé à diverses mesures industrielles

La disposition générale est celle d'un galvanomèrre d'Arsonval ordinaire. L'électro-aimant, en forme de couronne horızontale, est à pôles saullants intérieurs. Entre ces deux pôles se trouve placé le noyau de fer cylindrıque, également feuilleté. Les différentes parties de l'apparesl sont isolées à l'ebonite.

Les fuites magnétıques de l'électro-aimant alternatif induisent dans les pieces métalliques fixes de l'appareil des courants qui tendent à réagir sur le circunt du cadre. S'il y a quelque dissymétrie dans la construction, le cadre mobile sera donc legèrement entrainé d'un côté ou de l'autre comme dans un champ tournant. On ne peut pas farre qu'il n'y alt aucune dissymétrie, mais il est facile de supprimer, une fois pour toutes, l'effet d'entrainement dont 11 vient d'être question, en plaçant dans l'entrefer une petite lame de métal convenablement orientée.

Couple directeur electrıue. Sa suppression. - L'électro-aimant étant excite, s! l'on ferme le circuit du galvanomètre sur une résistance faible, on voit le cadre mobile se fixer violemment dans la position où 1 n'est traversé par aucun flux. S1 on l'écarte de cette position, il y est ramené par un couple directeur puissant qui s'ajoute au couple de torsion du fil. Il semble donc que la sensibilité de l'appareil doive se trouver énormément rédulte.

Ce couple directeur provient du courant induit dans le cadre par le flux alternatif. Si ce courant crait exactement en quadrature avec le champ, il ne produrait aucune dévation; le couple observé est dû au retard de phase que la self-induction du cadre impose au coulrant. Pour taire disparaitre le couple, il n'y a qu'à faire disparaître le retard de phase

Or, ceci est facile à ubtenir. On met en série avec le cadre mobile une capacitá shuntée par une résistance réglable. Cette deuxieme partie du circuit avance la phase du courant, et, pour une valeur convenable de la résistance, en fait disparaître le retard de phase et, en même temps, le couple directeur.

On peut remarquer que la compensation est ainsi faite une fois pour toutes, et que le circuit du cadre mobile, suvi de la capacité shuntée, n'intervient plu's que par sa résistance (1).

Sensibulité obtenue. - Le cadre mobile a, par exemple, une résistance de 200 ohms et une période d'oscillation de ro secondes. La compensation du couple directear est obtenue par la mise en circunt de un demi-microfarad shunté par une résistance de 330 ohms. Le

(1) Une capacité $C$ shuntée par une résistance $R$ compense une selfinduction $L$ donnee par la formule

$$
L=\frac{C R^{2}}{I+\omega^{2} C^{2} R^{2}}
$$

Si donc on met dans le circuit du cadre mobile une self-induction inconnue, la mesure de la resistance de réglage fera connaître la valeur de cetıe self-induction. I.e procedé est très commode pour la mesure à pour roo près les coefficients de self-induction, à partir de quelyues nililièmes de Henry. galvanomètre fermé sur une résistance de $2000 \mathrm{ohms,}$ est apériodque et son zéro est stable, à moins de un demi-millimètre près, l'échelle étant à un mètre de l'appareil. Dans ces conditions, la sen. sibulité est de 275 millimètres par micro-ampère.

Remarque. - Lorsque l'on fait le réglage de la compensation du couple directeur électrique, 11 peut être intéressant d'exagérer l'action de la capacité de réglage, de manière à mettre le courant induit dans le cadre en avance sur la force électro-motrice. Au lieu d'un counle directeur, on a alors un couple d'instabilité qui se retranche du couple de torsion du fil, et l'on arrive à ce résultat paradoxal d'obtenir une sensibilité plus grande que ne le comporte la torsion du fil.

Dans l'expérience précédente, par exemple, si l'on porte de 330 340 ohms la résistance qui shunte la capacité compensatrice, la sensibılité atteınt 400 millımètres par micro-ampère.

Sur les spectres des alliages. - Note de MM. de Kowalski et P.-B. Huber, séance du 30 avril $r g 06$.

C'est en étudiant l'influence de la self-induction sur les spectres de la decharge oscillante, entre des électrodes en alliages métallıues, que nous avons observé un phénomène qui nous paraìt assez intéressant pour être signalé On sait, d'après les recherches de Schuster et Hemsalech, qu'en intercalant une selt-induction dans le circuit de la decharge oscillante d'un condensateur, on fait disparaître dans le spectre de la décharge, non seulement les lignes de l'air, mais ausst certaines lignes de métal formant les électrodes.

Ce phénomèue s'explique, solt par l'abaissement de la température moyenne dans la décharge, soit en admettant avec J.-J. Thomson que l'émission de la lumière par un système corpusculaire faisant partie d'un atome n'a lieu que si la valeur de l'énergie intérieure du système dépasse un certain minimum. Si donc l'atome fait partie d'un milieu traversé par le courant électrique, comme cela a lien dans une décharge, cette énergte intérieure serait une fonction expo. nentielle du temps et des paramètres qui dépendent de l'intensité du courant, ainsı que des propriétés du milieu dans lequel se trcuve I'atome.

On pourrait donc s'attendre à ce que l'influence de la self-induction pût se manifester d'une façon différente selon que les électrodes seraient en métal pur ou bien en allage, et cela parce que le mileu ambiant du système émettant la lumsère est different dans les deux cas.

les faits que nous avons observés confirment cette prévision.

Notre circult de décharge était composé : 10 d'une batterie de condensateurs ayant une capacité d'environ 0,003 microfarad t d'un crrcuit très peu inductit formé du déchargeur et de fils qui le reliaient aux condensateurs; $2^{\circ}$ de la même batterie de condensấteurs, plus une bobine d'induction, sans noyau de fer, ayant une valeur de self-induction d'environ o, I henry. La distance explosive était dans toutes les expériences égale à $8 \mathrm{~mm}$.

Les photographies du spectre ont été faltes avec un spectrographe construit sur les indications de l'un de nous et possédant une lentille de Cornu en quartz, ainsi que des objectifs achromatısés par la combinaison des lentilles en quartz et en spathfluor. On obtenatt ainsi sur une seule plaque des photographies irès nettes dans toute l'étendue du spectre, depuis les raies vertes jusqu'aux raies ultıaviolettes de Cornu (environ $2000 \mathrm{~A}$ ).

Sans la self-1nduction, le temps de pose était de 30 secondes. Avec la self-induction, la pose durait toujours dix minutes. Les alliages que nous avons ctudiés étaient le cuivre-magnésium et le cuivrezinc; nous avons étudié également les métaux purs entrant dans la composition de ces alliages.

L'étude des photographies de spectres nous permet de résumer les faits de la façon suivante :

I. En intercalant la self-induction dans le circuit de la décharge, on falt disparaitre, dans le cas des électrodes en métal pur, un plus grand nombre de lignes du spectre que dans la cas des élecirodes en allage.

2. Les lignes qui n'ont pas disparu dans le spectre des alliages sont les mêmes pour l'allage du cuivre-zinc que du cuivre-magnésium et appartiennent au cuivre ;

3 . Leurs longueurs d'onde exprimées en unités Angström, sont les suivantes:

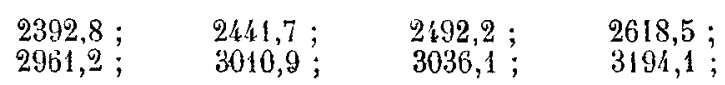

4. Toutes ces lignes ont été observées dans le spectre de l'arc par Kayser et Runge. sauf toutefors la ligne 2824,5 . Dans nos photogiaphies, cette ligne est très faible, mais peut être mesurée avec précjsion.

Les phénomènes décrits, et qui peuvent être, comme nous l'avons dit, déduits des idées de J. J. Thomson, pourraient cependant êre aussi expliqués en admetrant que la température moyerne dans la décharge oscillante entre électrodes en alliage est supérieure à celle qui existe dans la décharge analogue entre électrodes en cuivre pur: Il faudrait pour cela admetre que la température de vaporisation de l'allıage est supérıure à celle du métal pur. 